\title{
Comparison of inhalation technique with the Diskus and Autohaler in asthmatic children at home
}

\author{
Annelies van der Kolk ${ }^{1}$, Natasja Lammers (i) $^{2}$, Marjolein Brusse-Keizer ${ }^{3}$, \\ Job van der Palen ${ }^{3,4}$, Joyce Faber ${ }^{1}$, Reina Spenkelink-Visser ${ }^{5}$ and \\ Bernard J. Thio ${ }^{2,3}$
}

Affiliations: ${ }^{1}$ Dept of Pediatrics, Deventer Ziekenhuis, Deventer, The Netherlands. ${ }^{2}$ Dept of Pediatrics, Medisch Spectrum Twente, Enschede, The Netherlands. ${ }^{3}$ Medical School Twente, Medisch Spectrum Twente, Enschede, The Netherlands. ${ }^{4}$ Dept of Research Methodology, Measurement and Data Analysis, University of Twente, Enschede, The Netherlands. ${ }^{5}$ Dept of Pediatrics, Gelre Ziekenhuizen, Apeldoorn, The Netherlands.

Correspondence: Natasja Lammers, Dept of Pediatrics, Medisch Spectrum Twente, Koningsplein 1, Enschede 7500 KA, the Netherlands. E-mail: n.t.lammersagmail.com

\section{ABSTRACT}

Objective: Asthma is the most common chronic disease in childhood and anti-inflammatory medication is the cornerstone of treatment. Inhalers are frequently used incorrectly when demonstrated in the hospital, suggesting poor technique at home. We aimed to 1) compare daily inhalation technique with the Diskus and Autohaler in asthmatic children by filming inhalations at home and 2) compare daily inhalation technique with technique demonstrated in the hospital.

Methods: We performed a randomised study in asthmatic children (aged 6-18 years) from the outpatient clinic of Medisch Spectrum Twente hospital (Enschede, The Netherlands) from July 2014 to April 2016. Children received inhalation instructions for the Diskus and Autohaler and were randomised to use one device in the morning and the other in the evening. During the 28-day study period, inhalations were filmed at home and subsequently demonstrated in the hospital. All inhalations were checked for seven critical errors per device.

Results: 636 videos with the Diskus and 663 with the Autohaler were provided by 27 children. The most common critical error in daily life was an incorrect device position during preparation of the Diskus $(n=271)$ and an insufficiently deep inhalation $(n=39)$ using the Autohaler. Percentage of correct days using the Diskus was $44 \%$, compared to $96 \%$ with the Autohaler $(\mathrm{p}<0.001)$. The two most common errors with the Diskus were made at least twice as often at home than in the hospital.

Conclusion: Inhalation technique at home was markedly better with the Autohaler than with the Diskus. Paediatricians should be aware that hospital-based demonstrations can overestimate daily inhalation technique with the Diskus.

\section{@ERSpublications}

Inhalation technique at home is markedly better with the Autohaler than with the Diskus. Paediatricians should be aware that hospital-based demonstrations can overestimate daily inhalation technique with the Diskus. https://bit.ly/3cYK4eq

Cite this article as: Van der Kolk A, Lammers N, Brusse-eizer M, et al. Comparison of inhalation technique with the Diskus and Autohaler in asthmatic children at home. ERJ Open Res 2021; 7: 00215-2019 [https://doi.org/10.1183/23120541.00215-2019].

This study is registered at www.trialregister.nl with identifier number NL4302. Individual participant data that underlie the results reported in this article will be available, after deidentification (text, tables and figures), as well as the study protocol (including a statistical analysis plan), informed consent forms and analytic code, immediately following publication until 36 months after publication, with investigators whose proposed use of the data has been approved by an independent review committee, identified for this purpose, to achieve aims in the approved proposal. Proposals should be directed to n.t.lammers@gmail.com. To gain access, data requestors will need to sign a data access agreement after the approval of the independent review committee. After 36 months the data will be available from our hospital warehouse but without investigator support.

Received: 23 Aug 2019 | Accepted after revision: 4 Feb 2021

Copyright $\odot$ The authors 2021. This version is distributed under the terms of the Creative Commons Attribution NonCommercial Licence 4.0. For commercial reproduction rights and permissions contact permissions@ersnet.org 


\section{Introduction}

Asthma is the most common chronic disease in childhood. It is characterised by chronic airway inflammation and reversible airway obstruction with an increased airway responsiveness to a variety of stimuli [1]. Daily anti-inflammatory medication is the cornerstone of treatment and can effectively reduce airway inflammation and control symptoms [2]. The inhaled route is preferred as it minimises systemic side-effects while maintaining efficacy.

Commonly used devices for the inhalation of medication in childhood asthma are pressurised metered-dose inhalers (pMDIs) and dry-powder inhalers. Breath-actuated pMDIs such as the Autohaler and Redihaler incorporate a mechanism activated during inhalation that triggers the metered-dose inhaler. In dry-powder inhalers such as the Diskus and Turbuhaler the drug is dispersed into particles by inspiration [3].

Optimal pulmonary deposition of inhaled medication is highly dependent on a correct inhalation technique. Several studies have shown that inhalers are frequently used incorrectly, leading to poor asthma control [4-7]. A recent study conducted in a group of children hospitalised for asthma [7] found that almost half the participants demonstrated improper inhaler use in the hospital. Kamps et al. [8] showed that comprehensive inhalation instructions are needed to attain a correct, hospital-observed inhalation technique. However, inhalation technique demonstrated in front of healthcare professionals may overestimate daily technique at home. As yet, there are no studies focusing on the daily inhalation technique of children at home, which is vital information when attempting to improve asthma control. Therefore, we compared the daily inhalation technique with the Autohaler and the Diskus by filming inhalations at home and compared daily technique at home with technique as demonstrated in the hospital.

\section{Methods}

\section{Study design and patients}

This study had a randomised design. From July 2014 to April 2016, children aged 6-18 years with a clinical history of asthma from the paediatric outpatient department of Medisch Spectrum Twente (Enschede, the Netherlands) were asked to participate. Patients were eligible when they already used inhaled steroids twice daily (using a non-breath-actuated pMDI with spacer) or when their paediatrician was planning to start treatment with inhaled steroids.

\section{Study procedure}

Baseline visit

At baseline visit, a medical history with a specific focus on asthmatic symptoms and a physical examination was carried out. Patients were randomly assigned to treatment group 1 or treatment group 2, using opaque sealed envelopes with a randomisation number. Group 1 had to inhale one puff of fluticasone propionate $100 \mu \mathrm{g}$ using the Diskus in the morning and one puff of beclometasone dipropionate $100 \mu \mathrm{g}$ with the Autohaler in the evening, and group 2 vice versa. After this, patients were instructed on how to use the Diskus and the Autohaler by an experienced nurse practitioner, following standardised inhalation protocols of the Lung Alliance Netherlands (LAN) [9]. Patients had to demonstrate a correct inhalation technique for both devices after receiving the instructions. This was checked using the Inhalation Manager, a computer-based measuring instrument which enables testing the entire inhalation manoeuvre of commonly used breath-actuated inhalers [10].

\section{Filming inhalation technique at home}

Over a study period of 28 days, patients or parents filmed the inhalation of their medication at home twice daily using an iPad and were instructed to submit the video clips at the end of each day. In addition, they were instructed to inform the investigators when they experienced side-effects. An electronic reminder was sent to the iPad the following day if video clips were not received. Patients were excluded if video clips were not received more than three times in one of the four study weeks or more than three consecutive days. Video clips were scored according to a list of critical errors, partly adapted from the standardised inhalation protocols distributed by the LAN [9] (table 1). Critical errors were defined as errors that compromised the potential benefit of treatment, such as those that impede pulmonary drug deposition or delivery of a sufficient dose. All video clips were scored by the same investigator within 2 weeks after the last patient completed the study protocol.

Follow-up visit

At the end of the study period, patients visited the hospital for a live demonstration of their inhalation technique, performed in front of the investigator who scored their inhalation videos, using the same list of critical errors (table 1). 


\section{TABLE 1 List of critical errors used for the scoring of inhalation technique ${ }^{\ddagger}$}

Diskus

Autohaler

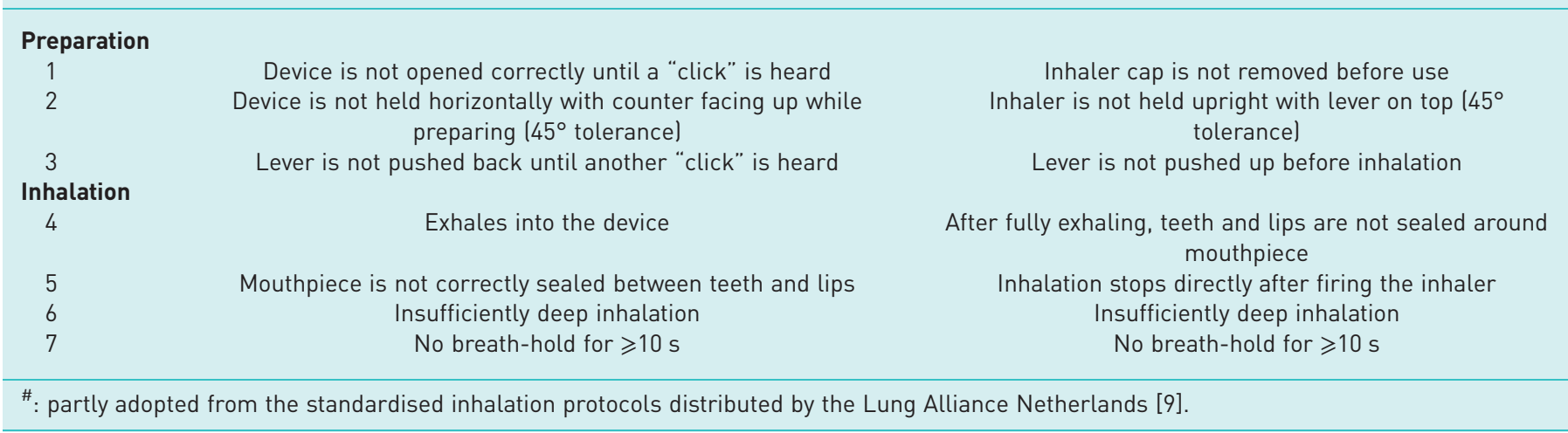

\section{Statistical analyses}

Results were expressed as mean $\pm \mathrm{SD}$ for the normally distributed continuous data and as median (interquartile range (IQR)) for non-normally distributed data. Normality was tested by viewing normality plot and histograms. For nominal or ordinal data, numbers with corresponding percentages were used.

Differences in baseline characteristics between the study groups were tested with the Mann-Whitney U-test for the non-normally distributed variables and the independent t-test for the normally distributed variables. Differences between binary variables were tested using the Chi-squared test or Fisher exact test, as appropriate. The Wilcoxon signed-rank test was used to test the difference between the percentage of correct inhalations for both devices at home and for the median amounts of critical errors per device per patient. Time until the first critical error was analysed with the log-rank test and visualised with a Kaplan-Meier plot. The McNemar test was used to analyse the percentages of patients who showed a correct inhalation technique during demonstration in the hospital.

A two-sided p-value $<0.05$ was considered statistically significant. Data analyses and the random allocation sequence were performed with SPSS Statistics, version 22.0.

\section{Ethical considerations}

This study was approved by the medical ethics review board Twente and registered in the Dutch trial register (NTR, identification NL4302). All children and parents/guardians received written patient information and provided written informed consent before participating in the study.

\section{Results}

Out of the 32 patients initially included in the study, five were excluded from further analyses because video clips were not received more than three times in 1 week or not received on more than three consecutive days. The 27 remaining patients provided 1299 video clips; 636 with the Diskus and 663 with the Autohaler, which were all suitable for analysis.

\section{Characteristics of the study population}

The baseline characteristics of the initial study sample $(n=32)$ are shown in table 2 . The initial study group comprised $20(62.5 \%)$ boys and $12(37.5 \%)$ girls with a mean \pm sD age of $7.9 \pm 1.7$ years. After randomisation, 16 patients were included in group 1 (Diskus - Autohaler) and 16 in group 2 (Autohaler Diskus). No differences in baseline characteristics nor in inhalation technique at home or in the hospital between the groups were observed (table 2 and not shown). Accordingly, data are presented for the study group as a whole.

\section{Inhalation technique at home}

During the study period of 28 days, a total of 451 critical errors were made with the Diskus, compared to 63 errors with the Autohaler. All patients made at least one critical error with the Diskus during the study period, while eight (29.6\%) patients made no critical error using the Autohaler.

The most common error with the Diskus in daily life was not holding the device horizontally with the counter facing upwards while preparing the dose before inhalation ( $\mathrm{n}=271 ; 60 \%$ of errors with the Diskus), followed by an insufficiently deep inhalation $(n=83 ; 18 \%)$ and exhaling into the device $(n=66 ; 15 \%)$ (table 3$)$. 


\begin{tabular}{|c|c|c|c|c|}
\hline & Group $1^{\#}$ & Group 2" & Total & p-value \\
\hline Patients & 16 & 16 & 32 & \\
\hline Sex & & & & 0.144 \\
\hline Female & $8(50)$ & $4(25)$ & 12 (37.5) & \\
\hline Male & $8(50)$ & $12(75)$ & 20 (62.5) & \\
\hline Age years & $8.0 \pm 1.6$ & $7.8 \pm 1.8$ & $7.9 \pm 1.7$ & 0.977 \\
\hline Atopy & 14 (87.5) & 13 (81.3) & 27 (84.4) & 1.000 \\
\hline \multicolumn{5}{|c|}{ Medication use } \\
\hline SABA & 14 (87.5) & 15 (93.8) & $29(90.6)$ & 1.000 \\
\hline LABA & 2 (12.5) & 2 (12.5) & 4 (12.5) & 1.000 \\
\hline ICS & 11 (68.8) & 15 (93.8) & 26 (81.3) & 0.172 \\
\hline NCS & 9 (56.3) & 5 (31.3) & $14(43.8)$ & 0.143 \\
\hline LTRA & 2 (12.5) & 2 (12.5) & 4 (12.5) & 1.000 \\
\hline \multicolumn{5}{|c|}{ 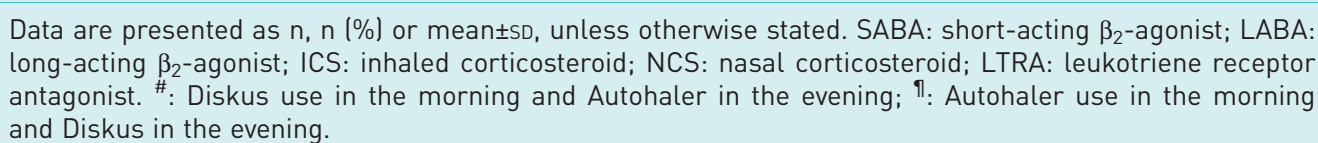 } \\
\hline
\end{tabular}

The most common error using the Autohaler was an insufficiently deep inhalation $(n=39 ; 62 \%$ of errors with the Autohaler), followed by an insufficient breath-hold after inhalation ( $n=18 ; 29 \%)$.

With the Diskus, the first error was made after a median (IQR) 2 (1-3) days, compared to 5 (4-14) days with the Autohaler $(\mathrm{p}<0.001)$. Figure 1 shows the survival function of patients without a critical error during the study period, showing a more rapid decline when using the Diskus.

The percentage of correct inhalations at home was $44 \%$ with the Diskus, compared to $96 \%$ with the Autohaler $(\mathrm{p}<0.001)$ (table 4). The median (IQR) number of critical errors made per patient was significantly higher using the Diskus $(14,10-22)$, compared to the Autohaler $(1,0-20)(\mathrm{p}<0.001)($ table 4$)$.

\section{Inhalation technique in the hospital}

During the demonstration in the hospital at the end of the study period, 19 (70\%) children showed a correct inhalation technique with the Diskus, and 23 (85\%) children showed a correct inhalation technique with the Autohaler $(\mathrm{p}<0.001)$ (table 4).

In figure 2, the prevalence of the two most common critical errors for each device in the home situation and during demonstration in the hospital is presented. The two most common errors with the Diskus (incorrect position of device and insufficiently deep inhalation) were made more than twice as frequently at home than in the hospital. The two most common errors with the Autohaler (insufficiently deep inhalation and insufficient breath-hold) were made slightly more frequently in the hospital than at home.

TABLE 3 Prevalence of different critical errors with the Diskus and Autohaler at home $(n=27)^{\#}$

\begin{tabular}{ccc} 
& Diskus & Autohaler \\
\hline Critical error type $^{\text {q }}$ & & \\
1 & $0(0)$ & $0(0)$ \\
2 & $271(60.1)$ & $4(6.3)$ \\
3 & $3(0.7)$ & $0(0)$ \\
4 & $66(14.6)$ & $1(1.6)$ \\
5 & $0(0)$ & $1(1.6)$ \\
6 & $83(18.4)$ & $39(61.9)$ \\
7 & $28(6.2)$ & $18(28.6)$ \\
Total & $451(100)$ & $63(100)$
\end{tabular}

Data are presented as $\mathrm{n}(\%) .{ }^{\#}$ : analyses are based on 636 video clips with the Diskus and 663 with the Autohaler, made by 27 patients; $^{~}$ : critical error types are described in table 1. 


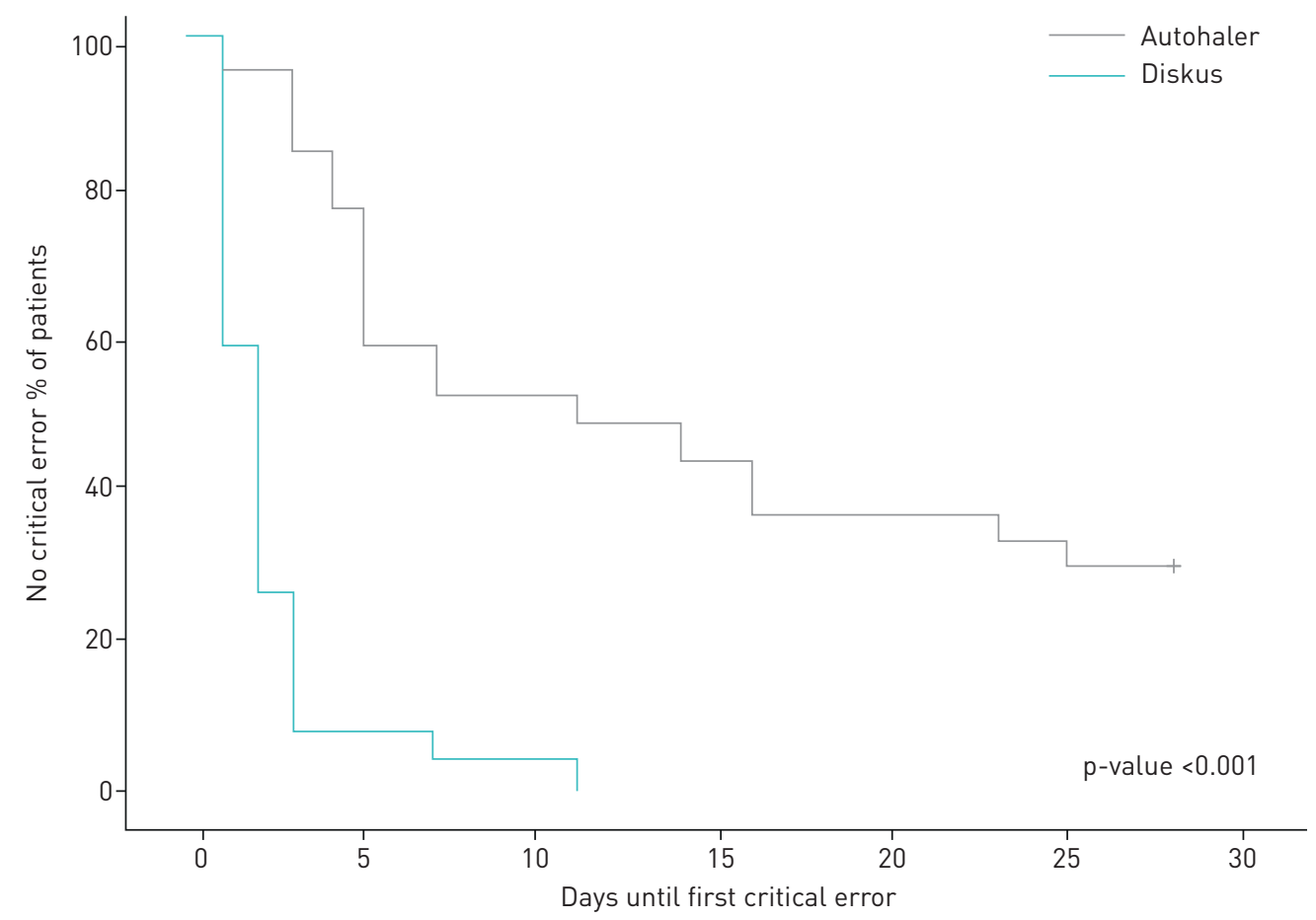

FIGURE 1 Survival function of percentage of patients without a critical error during the study period of 28 days, using the Diskus and Autohaler.

\section{Age differences}

Exploratory analyses were performed in two different age groups (6-7 years and 8-12 years) (data not shown). Children in the younger age group made more errors compared to the older age group regarding deep inspiration and breath-hold. This was observed for both devices, although it was more pronounced in the Autohaler group. In both age groups the demonstrated inhalation technique in the hospital with the Diskus overestimated the daily technique at home.

\section{Discussion}

We found that the inhalation technique with the Autohaler was well maintained after inhalation instructions, as in $96 \%$ of the study days no critical errors were made with this device. In contrast, we observed that the quality of medication administration with the Diskus declined rapidly after receiving instructions. Worryingly, the daily technique with the Diskus was not well reflected by demonstration in the hospital, overestimating the technique at home.

To our knowledge, this is the first study to investigate the inhalation technique of asthmatic children by filming inhalations at home. Previous studies focused on inhalation technique demonstrated in a primary-care or hospital setting, where technique was assessed through video conferencing [11] or directly in front of healthcare providers and caregivers.

\section{TABLE 4 Inhalation technique at home and during demonstration in the hospital with the}

\section{Diskus and Autohaler $(\mathrm{n}=27)^{\#}$}

\begin{tabular}{lccc} 
& Diskus & Autohaler & p-value \\
\hline Correct inhalations at home ${ }^{\pi} \%$ & $44.0(20.8-57.1)$ & $95.8(87.5-100)$ & $<0.001$ \\
Critical errors per patient $_{\text {Correct inhalation technique in the hospital }}{ }^{+}$ & $14(10-22)$ & $1(0-3)$ & $<0.001$ \\
& $19(70.4)$ & $23(85.2)$ & $<0.001$
\end{tabular}

Data are presented as median (interquartile range) or $\mathrm{n}(\%)$, unless otherwise stated. \#: analyses are based on 636 video clips with the Diskus and 663 with the Autohaler made by 27 patients; ${ }^{~}$ : percentage of inhalations without a critical error; ${ }^{+}$: patients who showed a correct inhalation technique during demonstration in the hospital at the end of the study period of 28 days. 


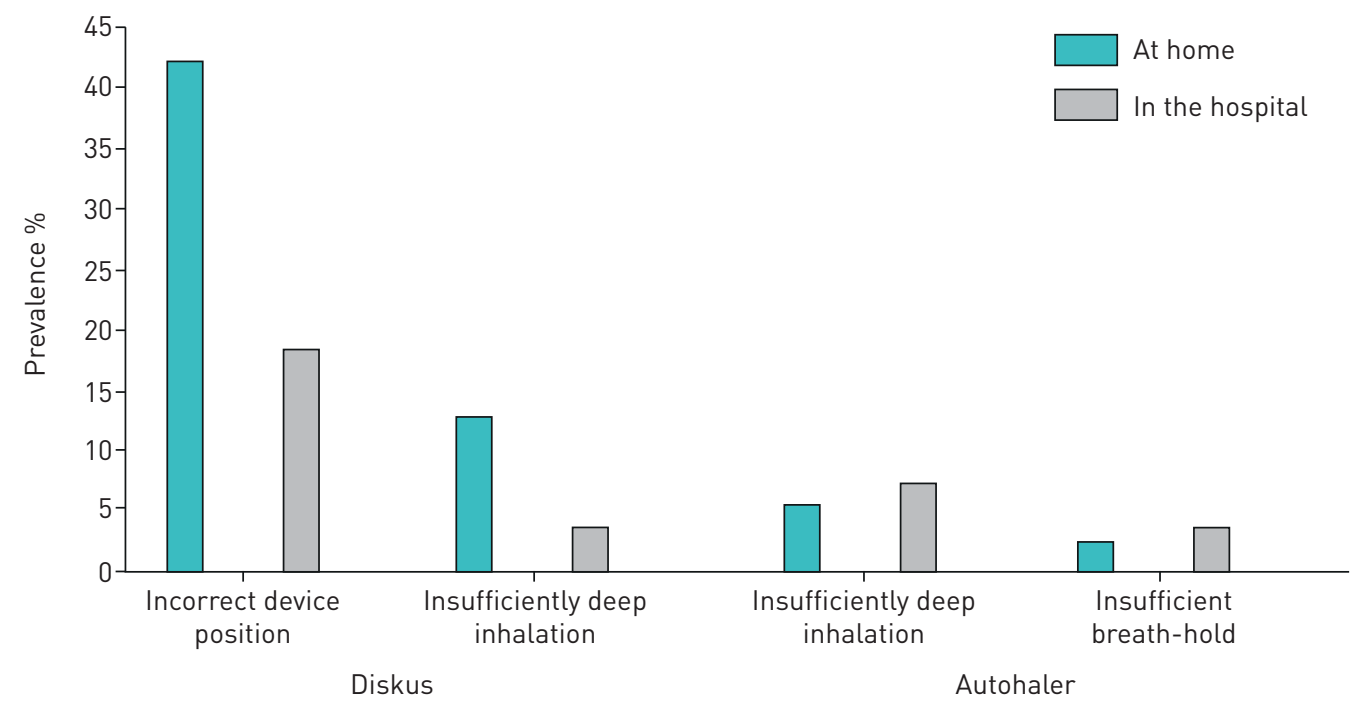

FIGURE 2 Prevalence of the two most common critical errors at home using the Diskus and Autohaler, compared to the prevalence at demonstration in the hospital, expressed as percentage of total observations ( $n=636$ for Diskus at home; $n=663$ for Autohaler at home; $n=27$ for both devices in the hospital).

Studies focusing on the inhalation technique of children using dry-powder inhalers show a wide range of correct use. In a study by SLEATH et al. [12], correct Diskus use was found in only $21.9 \%$ of the study population. CAPANOGLU et al. [5] found correct use in 34.6\% of their patients, comparable with the correct use of our study population at home. In a study by МАLOт et al. [13], primary-care physicians assessed the inhalation technique of children with their current device using device-specific checklists. They found that $46 \%$ of the population made at least one mistake with the Diskus.

KAMPs et al. [8] observed a correct inhalation technique with the Diskus in $79 \%$ of their study patients who had received instructions at a pharmacy, compared to $39 \%$ of the patients who had been trained by their general practitioner. In a control group that was trained at least twice by a research fellow during a 6-week period, 93\% showed a correct technique. Similarly to KAMPs et al., we provided comprehensive inhalation instructions consisting of both information and training of inhalation technique. The difference in correct inhalation technique after receiving instructions between our study and the study by KaMPs et al. could be due to the fact that the most common critical error in our study group was an incorrect positioning of the device during preparation; an error that was not taken into account in the aforementioned study. Additionally, the provision of repetitive inhalation instructions is likely to have had a positive effect on the sustainability of inhalation technique, as has been shown before by the same authors [14].

The most prevalent critical error in daily life with the Diskus was an incorrect preparation of the dose before inhalation; holding the device in a vertical position after opening the inhaler or holding the counter downwards. In the home situation this error was made during $42.5 \%$ of the inhalations. However, only five (18.5\%) out of 27 children showed this error in the hospital during demonstration. Another common critical error at home with the Diskus was not performing a deep inhalation (13.1\%). Only one child showed this error in the hospital. Apparently children are less focused on a correct inhalation technique at home compared to a demonstration in the hospital in front of healthcare professionals. It is to be expected that the technique of children who are not participating in a clinical trial and being filmed, would be even less accurate. The risk of overestimating the inhalation technique with the Diskus should therefore be taken into account by healthcare providers.

Technology-based methods for the monitoring of inhalation technique in general are promising. Several novel methods show good feasibility [11] and efficacy when it comes to maintaining a correct inhaler use [15]. Sulaiman et al. [16] used a device that focused on both adherence and inhalation technique, monitoring most critical errors. Although efficacy on healthcare outcomes has not yet been proven [17], these novel methods could reduce the burden of hospital visits focusing on inhalation technique [15]. There is a need for controlled studies investigating these new monitoring methods, focussing on both clinical outcomes and healthcare costs.

Although the Autohaler is a commonly used device, we only found one other study focusing on the inhalation technique with this device in a paediatric population. In the aforementioned study by MALOT 
et al. [13], 57\% of the children made at least one error and $8 \%$ one critical error using the Autohaler. Similar to our study, an insufficiently deep inhalation was scored as a critical error. However, the second most frequent mistake in our population, an insufficient breath-hold, was not scored as critical by MALOT et al., making comparison with our results difficult.

The two most common errors in our population were made slightly more frequent in the hospital than at home. However, due to the overall low prevalence of errors with the Autohaler, this comparison is less powerful for this device.

A recent systematic review by Usmani et al. [18] showed a large variation in how critical inhalation errors are defined for different inhalation devices. For the Autohaler as example, there is no consensus on whether to label an insufficient breath-hold as critical or noncritical, and how long this breath-hold should be. The large variation in how inhalation errors are labelled makes it difficult to compare studies and to create a clear overview of the magnitude of the problem. We agree with UsMANi et al. that there is need for a consensus on defining critical and noncritical errors.

In our study, we provided comprehensive inhalation instructions for the participants, based on a checklist with seven critical errors, adapted from an inhalation checklist from the LAN [9] and based on our expert opinion. Children were only included if their technique for both devices was correct following instructions. This way, we tried to create the same correct baseline technique for both devices. The same nurse practitioner provided the inhalation technique training for all participants and scored the filmed inhalations and the hospital demonstrations.

A limitation of our study is that children used two devices simultaneously, which could have led to an increase in errors per device. Conversely, it is plausible that by filming the inhalations at home the behaviour of the participants was positively influenced. Therefore, we suspect that in real life, inhalation technique for both devices will be worse than we showed in this study. As mentioned before, our checklist with critical errors was based on expert opinion and a widely used inhalation checklist in the Netherlands [14]. Our list with critical errors was not validated, and therefore our choice of different errors may be subject to discussion.

Proper administration of inhaled medication is essential for effective asthma treatment. This includes a correct preparation of the dose before inhalation, especially with the Diskus. Healthcare professionals should be aware of device-specific critical errors and should put emphasis on these possible errors during training of technique. We recommend the development of technological solutions focussing on the monitoring of inhalation technique and the provision of feedback in daily life, thereby hopefully reducing critical errors and optimising therapy.

In summary, inhalation technique at home was markedly better with the Autohaler than with the Diskus after receiving one inhalation instruction in the hospital. Healthcare professionals should be aware that hospital-based demonstrations can overestimate daily inhalation technique in children, but probably also in adults with asthma or COPD, especially when using the Diskus.

Conflict of interest: None declared.

Support statement: This study was supported by Stichting Pediatrisch Onderzoek Enschede (Pediatric Research Foundation Enschede). Funding information for this article has been deposited with the Crossref Funder Registry.

\section{References}

1 Global Initiative for Asthma (GINA). Global Strategy for Asthma Management and Prevention. 2017. Available from: www.ginasthma.org/

2 Barnes PJ. The role of inflammation and anti-inflammatory medication in asthma. Respir Med 2002; 96: Suppl A, S9-S15.

3 Brocklebank D, Ram F, Wright J, et al. Comparison of the effectiveness of inhaler devices in asthma and chronic obstructive airways disease: a systematic review of the literature. Health Technol Assess 2001; 5: 1-149.

4 Crompton GK, Barnes PJ, Broeders M, et al. The need to improve inhalation technique in Europe: a report from the Aerosol Drug Management Improvement Team. Respir Med 2006; 100: 1479-1494.

5 Capanoglu M, Dibek Misirlioglu E, Toyran M, et al. Evaluation of inhaler technique, adherence to therapy and their effect on disease control among children with asthma using metered dose or dry powder inhalers. J Asthma 2015; 52: 838-845.

6 Román-Rodríguez M, Metting E, Gacía-Pardo M, et al. Wrong inhalation technique is associated to poor asthma clinical outcomes. Is there room for improvement? Current Opin Pulm Med 2019; 25: 18-26.

7 Samady W, Rodriguez VA, Gupta R, et al. Critical errors in inhaler technique among children hospitalized with asthma. J Hosp Med 2019; 14: 361-365.

8 Kamps AW, van Ewijk B, Roorda RJ, et al. Poor inhalation technique, even after inhalation instructions, in children with asthma. Pediatr Pulmonol 2000; 29: 39-42.

9 Long Alliantie Nederland. Inhaler Use Protocols. Available from: https://inhalatorgebruik.nl/nl/protocollen/ Date last accessed: 11 April 2019. 
10 Kamin WE, Genz T, Roeder S, et al. The inhalation manager: a new computer-based device to assess inhalation technique and drug delivery to the patient. J Aerosol Med 2003; 16: 21-29.

11 Thomas R, Locke ER, Woo DM, et al. Inhaler training delivered by internet-based home videoconferencing improves technique and quality of life. Respir Care 2017; 62: 1412-1422.

12 Sleath B, Ayala G, Gillette C, et al. Provider demonstration and assessment of child device technique during pediatric asthma visits. Pediatrics 2011; 127: 642-648.

13 Malot L, Molimard M, Abouelfatah A, et al. Assessment of the handling of inhaler devices: an observational study of children in primary care. Arch Pediatr 2007; 14: 1190-1195.

14 Kamps AW, Brand PL, Roorda RJ. Determinants of correct inhalation technique in children attending a hospital-based asthma clinic. Acta Paediatr 2002; 91: 159-163.

15 Ammari WG, Al-Hyari N, Obeidat N, et al. Mastery of pMDI technique, asthma control and quality-of-life of children with asthma: a randomized controlled study comparing two inhaler technique training approaches. Pulm Pharmacol Ther 2017; 43: 46-54.

16 Sulaiman I, Seheult J, MacHale E, et al. Irregular and ineffective: a quantitative observational study of the time and technique of inhaler use. J Allergy Clin Immunol Pract 2016; 4: 900-909.

17 Himes BE, Leszinsky L, Walsh R, et al. Mobile health and inhaler-based monitoring devices for asthma management. J Allergy Clin Immunol Pract 2019; 7: 2535-2543.

18 Usmani OS, Lavaroni F, Marshall J, et al. Critical inhaler errors in asthma and COPD: a systematic review of impact on health outcomes. Respir Res 2018; 19: 10. 